\title{
Dik açılı dördün genlik modülasyonu ve L-kollu MRC tekniğine dayalı bir kablosuz haberleşme sisteminin Fisher-Snedecor F sönümlü kanallardaki performansi
}

\author{
Performance of a wireless communication system based on rectangular \\ quadrature amplitude modulation and L-branch MRC schemes over Fisher- \\ Snedecor $F$ fading channels
}

\author{
Nuri Kapucu, ${ }^{1, *}$ \\ ${ }^{1}$ Hitit Üniversitesi, Elektrik-Elektronik Mühendisliği Bölümü, 19030, Çorum Türkiye
}

\begin{abstract}
Özet
Bu çalışmada, iletim yöntemi olarak dik açılı dördün genlik modülasyonunu kullanan $L$ kollu en yüksek oranlı birleştirme alıcısına sahip bir kablosuz haberleşme sisteminin Fisher-Snedecor F sönümlenme koşulları altındaki ortalama sembol hata oranı analiz edilmiştir. Analiz, $L$ kollu en yüksek oranlı birleştirme tekniğine dayalı alıcıdaki toplam anlık işaret-gürültü oranının olasılık yoğunluk fonksiyonuna dayanarak yapılmıştır. Ele alınan sistemin ortalama sembol hata oranı için Gauss $Q$ fonksiyonunun yaklaşık bir ifadesi kullanılarak kapalı formda yaklaşık bir çözüm sunulmuştur. Daha sonra ise sistemin asimptotik ortalama sembol hata oran1 türetilmiştir. Sönümlenme parametresi $(m)$, gölgeleme parametresi $\left(m_{\mathrm{s}}\right)$, kol sayısı $(L)$ ve modülasyon seviyesi değiştirilerek ortalama sembol hata oranı farklı konfigürasyonlar için gösterilmiştir. Buna ek olarak, türetilen yaklaşık ifadenin analitik sonuçları ile ortalama sembol hata oranı integralinin nümerik hesaplanması ile elde edilen sonuçların yakın uyum içinde olduğu da gösterilmiştir. Asimptotik sonuçların ise yüksek işaretgürültü oranı bölgesinde diğer sonuçlar ile çok iyi uyum gösterdiği gözlenmiştir.
\end{abstract}

Anahtar kelimeler: Fisher-Snedecor F sönümlenmesi, En yüksek oranlı birleştirme, Ortalama sembol hata oranı

\section{Giriş}

Kablosuz haberleşmede karşılaşılan en önemli sorunlardan birisi sönümlenmedir. Bu olumsuz etki ile mücadele etmede en önemli çözüm ise işaretin birbirinden bağımsız sönümlenmeye maruz kalmış kopyalarının alıcıya ulaştırılması olan çeşitleme teknikleridir. Çeşitleme yapılan sistemlerde seçmeli birleştirme (selection combining, SC), eşit kazançlı birleştirme (equal gain combining, EGC) ve en yüksek oranlı birleştirme (maximum ratio combining, MRC) gibi alıcıda birleştirme teknikleri kullanılmaktadır. $\mathrm{Bu}$ teknikler arasında en optimum olan yöntem MRC tekniğidir $[1,2]$. En optimum yöntem olan MRC tekniğine dayalı alıcıların performansı çeşitli çalışmalarda analiz edilmiştir ve bazıları [3-8]'de verilmiştir. Jamal ve Hamid [3], masif

\begin{abstract}
In this study, average symbol error rate (ASER) of a wireless communication system which employs L-branch maximum ratio combining (MRC) at receiver and uses rectangular quadrature amplitude modulation (RQAM) as a transmission scheme is analyzed under Fisher-Snedecor F fading conditions. The analysis is conducted based on probability density function of total instantaneous signalto-noise ratio (SNR) at L-branch MRC receiver. A closedform approximate solution is presented for the ASER of the considered system by utilizing an approximate expression of Gaussian Q function. Then, asymptotic ASER of the system is derived. The ASER is illustrated for different configurations by varying fading parameter $(\mathrm{m})$, shadowing parameter $\left(\mathrm{m}_{\mathrm{s}}\right)$, number of branches $(\mathrm{L})$ and modulation level. In addition, it is also shown that analytical results of the derived approximate expression are close to the ones obtained by the numerical computation of the ASER integral. It is observed that the asymptotic results are in well agreement with other results at high SNR region.
\end{abstract}

Keywords: Fisher-Snedecor $\mathrm{F}$ fading, Maximum ratio combining, Average symbol error rate

çok girişli çok çıkışlı (multiple-input multiple-output, MIMO) sistemlerde MRC tekniğine dayalı alıcıların performansını işaret-gürültü+girişim oranı (signal-tointerference-plus-noise ratio, SINR) için olasılık yoğunluk fonksiyonu (probability density function, PDF) türeterek analiz etmiştir. [4]'te verilen çalışmada, işbirlikli kod bölmeli çoklu erişim sistemlerinde alıcıda birleştirme işlemi MRC tekniği ile gerçekleştirilmiştir. Benzer şekilde [5]'te ise $L$ kollu MRC alıcıların $\eta-\mu$ ve $\kappa-\mu$ sönümlü kanallardaki ortalama sembol hata oranı (average symbol error rate, ASER) başarımı için kapalı formda ifadeler önerilmiştir. [6]'da $M$-li faz kaydırmalı anahtarlama modülasyonu kullanılan ve MRC alıcıların bulunduğu sistemin ortalama sembol hata oranı $\kappa-\mu$ sönümlü kanallarda analiz edilmiştir.

\footnotetext{
* Sorumlu yazar / Corresponding author, e-posta / e-mail: nurikapucu@ hitit.edu.tr (N. Kapucu)

Geliș / Recieved: 19.04.2021 Kabul / Accepted: 03.06.2021 Yayımlanma / Published: 27.07.2021

doi: 10.28948/ngmuh.922679
} 
Bir başka çalışmada [7]'de MRC alıcıların iki dalga yayılmış güç (two wave diffused power, TWDP) sönümlü kanallarda kanal kapasitesi başarımı sunulmuştur. Liu vd. [8], veri sezimi ve kanal kestirimi için düşük çözünürlüklü analogdijital çeviricilerin kullanıldığı masif MIMO sistemlerin alıcı kısmında MRC yöntemini tercih ederek Rician sönümlü kanallarda performans analizi yapmışlardır.

Yukarıda bir kısmı [3-8]'de verilen çalışmalarda MRC alıcıların performansı çeşitli sönümlü kanallarda analiz edilmiştir. Yakın zamanda bu sönümlü kanal modellerinden farklı olarak, çokyollu yayılım ve gölgeleme etkilerini birleşik olarak iyi bir şekilde tanımlayan, matematiksel olarak analiz kolaylığı sağlayabilen Fisher-Snedecor F isimli bir sönümlenmeli kanal modeli [9]'da önerilmiştir. Önerilen bu kanal modeli için bina içi ve bina dışı ortamlarda deneysel veriler toplanmış ve bu veriler ile kanal modelinin doğruluğu ortaya konulmuştur. Deneysel veriler, bina içi ve bina dış1 ortamlarda $5.8 \mathrm{GHz}$ frekans bandında cihazdan cihaza (device-to-device, D2D) haberleşme için elde edilmiştir [9, 10].

Bugüne kadar Fisher-Snedecor $\mathrm{F}$ sönümlü kanallarda çeşitli haberleşme sistemlerinin performansları analiz edilmiştir ve bazıları [11-22]'de verilmiştir. [11]'de tek girişli tek çıkışlı bir sistemde sayısal modülasyonların Fisher-Snedecor F sönümlü kanallardaki performansı sunulmuştur. Bununla birlikte, çeşitli iletim teknikleri için Fisher-Snedecor F sönümlü kanalın kapasitesi [12]'de ele alınmıştır. [13, 14]'te sırasıyla tek girişli tek çıkışlı ve çok girişli tek çıkışl1 sistemlerin Fisher-Snedecor F sönümlü kanallardaki efektif kapasitesi için analitik ifadeler türetilmiştir. Badarneh vd. [15] Fisher-Snedecor F değişkenlerin çarpımı ve oransal çarpımını ele alarak kaskat F kanalında performans analizi ile spektrum paylaşıml ağların performans analizini ortaya koymuştur. [16]'da F sönümlü kanallarda SC alıcıların, [17-19]'da ise MRC alıcıların performansı sunulmuştur. [20]'de ise F sönümlü kanalların seviye geçiş oranı (level crossing rate) ve ortalama sönüm süresi (average fade duration) bulunmuştur. İki atlamalı röleli haberleșme sistemlerinin $F$ sönümlü kanallardaki servis-dışı kalma olasılığı, hata analizi, kanal kapasitesi ve efektif kapasitesi [21]'de türetilmiştir. [22]'de F dağılımlı rastgele değişkenlerin toplamı için çok değişkenli Fox- $H$ fonksiyonu cinsinden PDF önerilmiş ve önerilen ifade kullanılarak MRC alıcıların servis-dışı kalma olasılığı, efektif kapasitesi ve farklı iletim teknikleri için kanal kapasitesi performansı gösterilmiştir.

Bilindiği kadarıyla literatürde, dik açılı dördün genlik modülasyonu (rectangular quadrature amplitude modulation, RQAM) yöntemini kullanan $L$ kollu MRC alıcısına sahip bir kablosuz haberleşme sisteminin Fisher-Sendecor $F$ sönümlenmeli kanallardaki ASER performansını analiz eden bir çalışma bulunmamaktadır. RQAM modülasyonu band genişliği yönünden verimli olan genel bir modülasyon türüdür ve yüksek hızlı mobil iletişim ile mikrodalga iletişim alanında pratik uygulamalara sahip olan çok önemli bir iletim yöntemidir [23]. Bu motivasyon ile bu çalışmada, RQAM iletim yöntemini kullanan ve $L$ kollu MRC alıcısına sahip sistemlerin Fisher-Snedecor F sönümlü kanallarda ortalama sembol hata oranı (average symbol error rate,
ASER) için PDF'ye dayalı olarak kapalı formda yaklaşık bir ifade türetilmiştir. Türetilen ifade yaklaşık bir ifade olup Gamma fonksiyonu, Whittaker fonksiyonu gibi popüler paket programlarda yerleşik olarak bulunan fonksiyonlar içermektedir ve Matlab ile Mathematica gibi popüler yazılımlar kullanılarak nümerik olarak kolaylıkla hesaplatılabilmektedir. Daha sonra, ele alınan sistemin yüksek SNR bölgesindeki davranışını analiz etmek için asimptotik bir ASER ifadesi önerilmiştir. Ele alınan sistemin ASER performansı sönümlenme parametresi, gölgeleme parametresi, kol sayısı ve modülasyon seviyesinin farklı konfigürasyonları için gösterilmiştir.

\section{Sistem ve kanal modelleri}

$\mathrm{Bu}$ çalışmada ele alınan MRC birleştirme yöntemi Şekil 1 'de gösterilmektedir. $L$ adet farklı yoldan gelen işaretler alıcıda birleştirilmektedir. Buna göre $l$. yoldan gelen işaretin $\operatorname{anlık}$ SNR'si $\left(\gamma_{l}\right)$

$$
\gamma_{l}=\frac{h_{l}^{2} E_{s}}{N_{0}}
$$

olarak ifade edilir. Burada, $h_{l} l$. yolun sönümlenme katsayısı, $E_{s}$ sembol enerjisi ve $N_{0}$ ise $l$. yolun tek tarafl güç spektral yoğunluğudur.

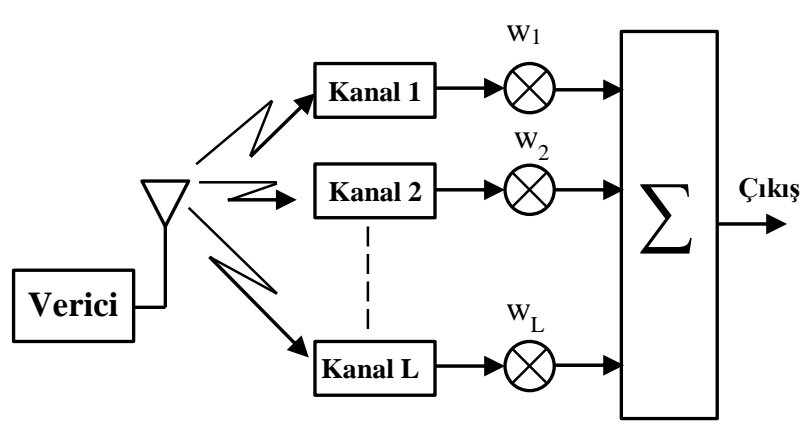

Şekil 1. MRC yöntemi blok şeması

Şekil 1'deki $w_{l}$ ifadesi $l$. yolun MRC yöntemindeki ağırlıklandırma katsayısıdır. $L$-kollu MRC alıcısında toplam anlık SNR aşağıdaki gibi ifade edilir:

$$
\gamma_{M R C}=\sum_{l=1}^{L} \gamma_{l}
$$

Şekil 1'deki $L$ adet kanal ise Fisher-Snedecor $F$ sönümlenmeli olarak kabul edilmiştir. Anlık SNR'ye ait PDF ifadesi Fisher-Snedecor F dağılımına göre

$$
f_{\gamma}(\gamma)=\frac{m^{m}\left(m_{s} \bar{\gamma}\right)^{m_{s}} \gamma^{m-1}}{B\left(m, m_{s}\right)\left(m \gamma+m_{s} \bar{\gamma}\right)^{m+m_{s}}}
$$


olarak verilir [9]. Burada, $m$ sönümlenme parametresi, $m_{s}$ gölgeleme parametresi, $B(\cdot, \cdot)$ ifadesi Beta fonksiyonunu göstermektedir. $\quad \bar{\gamma}$ ise ortalama $\mathrm{SNR}$ olup $\bar{\gamma}=\mathrm{E}[\gamma]$ şeklinde ifade edilir ve E[.] ise beklenen değerdir. Denklem (2)'ye dayalı olarak MRC alıcısındaki toplam anlık SNR için PDF ifadesi bağımsız ve özdeş dağılımlı $L$ adet FisherSnedecor F değişkeninin toplamına ait PDF'ye eşit olmaktadır. MRC alıcı çıkışındaki toplam anlık SNR için PDF aşağıdaki gibi ifade edilmektedir [18]:

$$
\begin{gathered}
f_{\gamma_{M R C}}(\gamma)=\frac{1}{\Gamma(m L)}\left(\frac{m}{m_{s} \bar{\gamma}}\right)^{m L}\left(\frac{\Gamma\left(m+m_{s}\right)}{\Gamma\left(m_{s}\right)}\right)^{L} \\
\times \gamma^{m L-1}{ }_{2} F_{1}\left(m+m_{s}, m L ; m L ;-\frac{m}{m_{s} \bar{\gamma}} \gamma\right)
\end{gathered}
$$

Burada, $L$ kol (ya da yol) sayısı, $\Gamma(\cdot)$ Gamma fonksiyonu, ${ }_{2} F_{1}(,, ; ; \cdot \cdot)$ ise Gauss hipergeometrik fonksiyondur.

\section{Ortalama sembol hata oranı analizi}

Ortalama sembol hata oranı şu şekilde ifade edilir:

$$
P_{s}(e)=\int_{0}^{\infty} P_{s}(e \mid \gamma) f_{\gamma}(\gamma) d \gamma
$$

$P_{s}(e \mid \gamma)$ ifadesi toplamsal beyaz Gauss gürültülü (additive White Gaussian noise, AWGN) kanalın koşullu sembol hata oranı (symbol error rate, SER) ve $f_{\gamma}(\gamma)$ ise kanal modeline ait anlı SNR için PDF ifadesidir. $M$-li RQAM tekniğinin AWGN kanalındaki koşullu SER ifadesi şu şekildedir:

$$
P_{s}(e \mid \gamma)=2\left[p Q\left(x_{1}\right)+q Q\left(x_{2}\right)-2 p q Q\left(x_{1}\right) Q\left(x_{2}\right)\right]
$$

Burada $Q(\cdot)$ sembolü Gauss $Q$ fonksiyonunu temsil etmektedir. Bunun yanında diğer parametreler; $M=M_{I} \times M_{Q}$ eş fazlı (in-phase) ve dördün (quadrature) elemanların çarpımından oluşan modülasyon seviyesi, $x_{1}=a \sqrt{\gamma}, \quad x_{2}=b \sqrt{\gamma}, \quad p=1-\left(1 / M_{I}\right), \quad q=1-\left(1 / M_{Q}\right)$, $b=\beta a, \beta=d_{Q} / d_{I}$ ve $a=\sqrt{\frac{6}{\left(M_{I}^{2}-1\right)+\left(M_{Q}^{2}-1\right) \beta^{2}}}$

şeklindedir. $d_{I}$ ve $d_{Q}$ parametreleri ise sırasıyla eş fazlı ve dördün karar uzaklıklarıdır [5]. Denklem (6)'daki ifade Denklem (5)'te yerine yazılarak ASER aşağıdaki gibi yazılır:

$$
\begin{aligned}
P_{s}(e) & =\int_{0}^{\infty} 2 p Q\left(x_{1}\right) f_{\gamma}(\gamma) d \gamma+\int_{0}^{\infty} 2 q Q\left(x_{2}\right) f_{\gamma}(\gamma) d \gamma \\
& -\int_{0}^{\infty} 4 p q Q\left(x_{1}\right) Q\left(x_{2}\right) f_{\gamma}(\gamma) d \gamma
\end{aligned}
$$

Bu integral Gauss $Q$ fonksiyonunu içerdiğinden bilindiği kadarıyla kapalı formda bir çözüme sahip değildir. $\mathrm{Bu}$ yüzden Gauss $Q$ fonksiyonunu üstel fonksiyonlar cinsinden ifade ederek Denklem (7)'deki integrale kapalı formda yaklaşık bir çözüm bulunabilir. Literatürde sıklıkla kullanılan ve Gauss Q fonksiyonunun iyi bir yaklaşıklığı olan ifade [24]'te önerilmiştir ve şu şekildedir:

$$
Q(x) \simeq \frac{1}{12} e^{-\frac{x^{2}}{2}}+\frac{1}{4} e^{-\frac{2 x^{2}}{3}}
$$

$\mathrm{Bu}$ yaklaşıklığın kullanılması ile Denklem (7)'deki integral için bir çözüm bulmak mümkün hale gelmiştir. Denklem (7)'deki ifade üç ayrı sembol ile aşağıdaki gibi yazılabilir:

$$
P_{s}(e)=I_{1}+I_{2}-I_{3}
$$

Denklem (4)'teki PDF ifadesinin kullanılması ile birlikte $I_{1}, I_{2}$ ve $I_{3}$ şu şekilde yazılır:

$$
\begin{aligned}
& I_{1}=2 p \int_{0}^{\infty}\left(\frac{1}{12} e^{-\frac{a^{2} \gamma}{2}}+\frac{1}{4} e^{-\frac{2 a^{2} \gamma}{3}}\right) \psi \gamma^{m L-1} \\
& \times{ }_{2} F_{1}\left(m+m_{s}, m L ; m L ;-\lambda \gamma\right) d \gamma \\
& I_{2}=2 q \int_{0}^{\infty}\left(\frac{1}{12} e^{-\frac{b^{2} \gamma}{2}}+\frac{1}{4} e^{-\frac{2 b^{2} \gamma}{3}}\right) \psi \gamma^{m L-1} \\
& \times{ }_{2} F_{1}\left(m+m_{s}, m L ; m L ;-\lambda \gamma\right) d \gamma \\
& I_{3}=4 p q \int_{0}^{\infty}\left(\frac{1}{12} e^{-\frac{a^{2} \gamma}{2}}+\frac{1}{4} e^{-\frac{2 a^{2} \gamma}{3}}\right)\left(\frac{1}{12} e^{-\frac{b^{2} \gamma}{2}}+\frac{1}{4} e^{-\frac{2 b^{2} \gamma}{3}}\right) \\
& \times \psi \gamma^{m L-1}{ }_{2} F_{1}\left(m+m_{s}, m L ; m L ;-\lambda \gamma\right) d \gamma
\end{aligned}
$$

Burada

$$
\psi=\frac{1}{\Gamma(m L)}\left(\frac{m}{m_{s} \bar{\gamma}}\right)^{m L}\left(\frac{\Gamma\left(m+m_{s}\right)}{\Gamma\left(m_{s}\right)}\right)^{L}
$$

ve $\lambda=m /\left(m_{s} \bar{\gamma}\right)$ olarak tanımlıdır. Denklem (10) ve Denklem (11)'de iki adet, Denklem (12)'de ise dört adet benzer formda integral bulunmaktadır. Bu integraller

$$
I(\rho)=\int_{0}^{\infty} \gamma^{m L-1} e^{-\rho}{ }_{2} F_{1}\left(m+m_{s}, m L ; m L ;-\lambda \gamma\right) d \gamma
$$


formundadır. Buna göre Denklem (10), Denklem (11) ve Denklem (12)'deki ifadeler Denklem (13)'e göre tekrar aşağıdaki gibi yazılır:

$$
\begin{gathered}
I_{1}=\frac{p}{6} \psi I\left(\frac{a^{2} \gamma}{2}\right)+\frac{p}{2} \psi I\left(\frac{2 a^{2} \gamma}{3}\right) \\
I_{2}=\frac{p}{6} \psi I\left(\frac{b^{2} \gamma}{2}\right)+\frac{p}{2} \psi I\left(\frac{2 b^{2} \gamma}{3}\right) \\
I_{3}=\frac{p q}{36} \psi I\left(\frac{a^{2} \gamma}{2}+\frac{b^{2} \gamma}{2}\right)+\frac{p q}{12} \psi I\left(\frac{a^{2} \gamma}{2}+\frac{2 b^{2} \gamma}{3}\right) \\
+\frac{p q}{12} \psi I\left(\frac{2 a^{2} \gamma}{3}+\frac{b^{2} \gamma}{2}\right)+\frac{p q}{4} \psi I\left(\frac{2 a^{2} \gamma}{3}+\frac{2 b^{2} \gamma}{3}\right)
\end{gathered}
$$

Buna göre Denklem (14)'teki integral için [25, 2.21.2.2]'den faydalanarak aşağıdaki ifade türetilmiştir:

$$
I_{1}=\frac{p}{6} \psi \Xi\left(\frac{a^{2}}{2}\right)+\frac{p}{2} \psi \Xi\left(\frac{2 a^{2}}{3}\right)
$$

Burada

$$
\begin{aligned}
& \Xi(\varphi)=\frac{\Gamma(m L)}{\varphi^{m L}}\left[\frac{\varphi}{\lambda}\right]^{\left(m+m_{s}+m L-1\right) / 2} e^{\frac{\varphi}{2 \lambda}} \\
& \times W_{\left(1-m-m_{s}-m L\right) / 2,\left(m+m_{s}-m L\right) / 2}\left(\frac{\varphi}{\lambda}\right)
\end{aligned}
$$

olarak türetilmiştir. Burada $W_{\text {., }}(\cdot)$ Whittaker fonksiyonudur. Türetilen bu ifade önce Denklem (14) ve benzer çözümler Denklem (15) ve Denklem (16)'da yerine yazılarak $I_{1}, I_{2}$ ve $I_{3}$ elde edilmiş ve daha sonra bu ifadeler Denklem (9)'da yerine yazılarak ASER türetilmiştir:

$$
\begin{aligned}
& P_{s}(e)=\psi p\left\{\frac{1}{6} \Xi\left(\frac{a^{2}}{2}\right)+\frac{1}{2} \Xi\left(\frac{2 a^{2}}{3}\right)\right\}+q \psi\left\{\frac{1}{6} \Xi\left(\frac{b^{2}}{2}\right)\right. \\
& \left.+\frac{1}{2} \Xi\left(\frac{2 b^{2}}{3}\right)\right\}-\psi p q\left\{\frac{1}{36} \Xi\left(\frac{a^{2}+b^{2}}{2}\right)+\frac{1}{12} \Xi\left(\frac{a^{2}}{2}+\frac{2 b^{2}}{3}\right)\right. \\
& \left.+\frac{1}{12} \Xi\left(\frac{2 a^{2}}{3}+\frac{b^{2}}{2}\right)+\frac{1}{4} \Xi\left(\frac{2 a^{2}}{3}+\frac{2 b^{2}}{3}\right)\right\}
\end{aligned}
$$

\section{Asimptotik analiz}

$\bar{\gamma} \rightarrow \infty$ iken Denklem (4)'teki PDF, [26, 07.23.03.0001.01]'deki özellik kullanılarak asimptotik olarak aşağıdaki gibi elde edilmiştir:

$$
f_{\gamma_{M R C}}^{\text {asymp }}(\gamma)=\frac{1}{\Gamma(m L)}\left(\frac{m}{m_{s} \bar{\gamma}}\right)^{m L}\left(\frac{\Gamma\left(m+m_{s}\right)}{\Gamma\left(m_{s}\right)}\right)^{L} \gamma^{m L-1}
$$

Denklem (20)'de verilen asimptotik PDF ifadesi kullanılarak $I_{1}, I_{2}$ ve $I_{3}$ asimptotik olarak şu şekilde yazilir:

$$
\begin{gathered}
I_{1}^{\text {asymp }}=2 p \int_{0}^{\infty}\left(\frac{1}{12} e^{-\frac{a^{2} \gamma}{2}}+\frac{1}{4} e^{-\frac{2 a^{2} \gamma}{3}}\right) \psi \gamma^{m L-1} d \gamma \\
I_{2}^{\text {asymp }}=2 q \int_{0}^{\infty}\left(\frac{1}{12} e^{-\frac{b^{2} \gamma}{2}}+\frac{1}{4} e^{-\frac{2 b^{2} \gamma}{3}}\right) \psi \gamma^{m L-1} d \gamma \\
I_{3}^{\text {assmp }}=4 p q \int_{0}^{\infty}\left(\frac{1}{12} e^{-\frac{a^{2} \gamma}{2}}+\frac{1}{4} e^{-\frac{2 a^{2} \gamma}{3}}\right)\left(\frac{1}{12} e^{-\frac{b^{2} \gamma}{2}}+\frac{1}{4} e^{-\frac{2 b^{2} \gamma}{3}}\right) \\
\times \psi \gamma^{m L-1} d \gamma
\end{gathered}
$$

[27, (3.381.4)]'teki ilişkinin yardımıyla $I_{1}^{\text {asymp }}, I_{2}^{\text {asymp }}$ ve $I_{3}^{a s y m p}$ için aşağıdaki ifadeler elde edilmiştir:

$$
\begin{aligned}
I_{1}^{\text {asymp }} & =p\left\{\frac{\psi}{6}\left(\frac{a^{2}}{2}\right)^{-m L} \Gamma(m L)+\frac{\psi}{2}\left(\frac{2 a^{2}}{3}\right)^{-m L} \Gamma(m L)\right\} \\
I_{2}^{\text {asymp }} & =q\left\{\frac{\psi}{6}\left(\frac{b^{2}}{2}\right)^{-m L} \Gamma(m L)+\frac{\psi}{2}\left(\frac{2 b^{2}}{3}\right)^{-m L} \Gamma(m L)\right\} \\
I_{2}^{a s y m p} & =p q \Gamma(m L)\left\{\frac{\psi}{36}\left(\frac{a^{2}+b^{2}}{2}\right)^{-m L}+\frac{\psi}{12}\left(\frac{3 a^{2}+4 b^{2}}{6}\right)^{-m L}\right. \\
& \left.+\frac{\psi}{12}\left(\frac{4 a^{2}+3 b^{2}}{6}\right)^{-m L}+\frac{\psi}{4}\left(\frac{2 a^{2}+2 b^{2}}{3}\right)^{-m L}\right\}
\end{aligned}
$$

Ele alınan sistemin asimptotik ASER ifadesi şu şekilde yazilır: $P_{s}^{\text {asymp }}(e)=I_{1}^{\text {asymp }}+I_{2}^{\text {asymp }}-I_{3}^{\text {asymp }}$

$$
\begin{aligned}
P_{s}^{a s y m p}(e) & =p\left\{\frac{\psi}{6}\left(\frac{a^{2}}{2}\right)^{-m L} \Gamma(m L)+\frac{\psi}{2}\left(\frac{2 a^{2}}{3}\right)^{-m L} \Gamma(m L)\right\} \\
& +q\left\{\frac{\psi}{6}\left(\frac{b^{2}}{2}\right)^{-m L} \Gamma(m L)+\frac{\psi}{2}\left(\frac{2 b^{2}}{3}\right)^{-m L} \Gamma(m L)\right\} \\
& -p q \Gamma(m L)\left\{\frac{\psi}{36}\left(\frac{a^{2}+b^{2}}{2}\right)^{-m L}+\frac{\psi}{12}\left(\frac{3 a^{2}+4 b^{2}}{6}\right)^{-m L}\right. \\
& \left.+\frac{\psi}{12}\left(\frac{4 a^{2}+3 b^{2}}{6}\right)^{-m L}+\frac{\psi}{4}\left(\frac{2 a^{2}+2 b^{2}}{3}\right)^{-m L}\right\}
\end{aligned}
$$

\section{Nümerik sonuçlar}

$\mathrm{Bu}$ bölümde ele alınan sistemin ASER performansını göstermek için çeşitli nümerik sonuçlar sunulmuştur. Denklem (19)'da önerilen yaklaşık ifadeden elde edilen analitik sonuçlar, Denklem (7)'deki integrallerin nümerik 
olarak hesaplatılması ile elde edilen tam sonuçlar ve Denklem (27)'deki asimptotik ifadeden elde edilen sonuçlar kıyaslamalı olarak gösterilmiştir.

Şekil 2'de ele alınan $L$-kollu MRC alıcılı sistemde 4X2 RQAM yöntemi kullanıldığı durum için alıcıdaki kol sayısına göre Fisher-Snedecor $F$ sönümlenmeli kanallardaki ASER performansı verilmiştir. Bu şekilde kanal sönümlenme parametresi $m=2$, gölgeleme parametresi $m_{\mathrm{s}}=2$ ve $\beta=1$ olarak alınmıştır. Şekil 2 incelendiğinde beklenildiği gibi kol sayısı arttığında performans iyileşmektedir. Dolayısıyla bu şekilden de görüleceği üzere $L=3$ olduğunda en iyi performans elde edilmiştir. Çeşitlemedeki amaç orijinal bilginin birbirinden farklı sönümlenmeye maruz kalmış kopyalarının alıcıda birleştirilmesi olduğundan dolayı fazla sayıda birbirinden bağımsız işaret alınması performansı iyileştirmektedir ve aynı zamanda bu durum alıcıda MRC birleştirmesi sonucunda elde edilen işaretin orijinal işarete yakın olması anlamına gelmektedir. Bunun yanında Denklem (17)'de önerilen yaklaşık ifadeden bulunan yaklaşık sonuçların Denklem (7)'nin nümerik olarak hesaplanması ile elde edilen tam sonuçlara oldukça yakın olduğu görülmektedir. $\mathrm{Bu}$ sonuç, önerilen yaklaşık ifadenin ele alınan sistem için iyi bir üst sınır olduğunu göstermektedir. Ayrıca Denklem (27)'den elde edilen asimptotik sonuçlar ise yüksek SNR bölgesinde diğer sonuçlar ile mükemmel uyum göstermektedir.

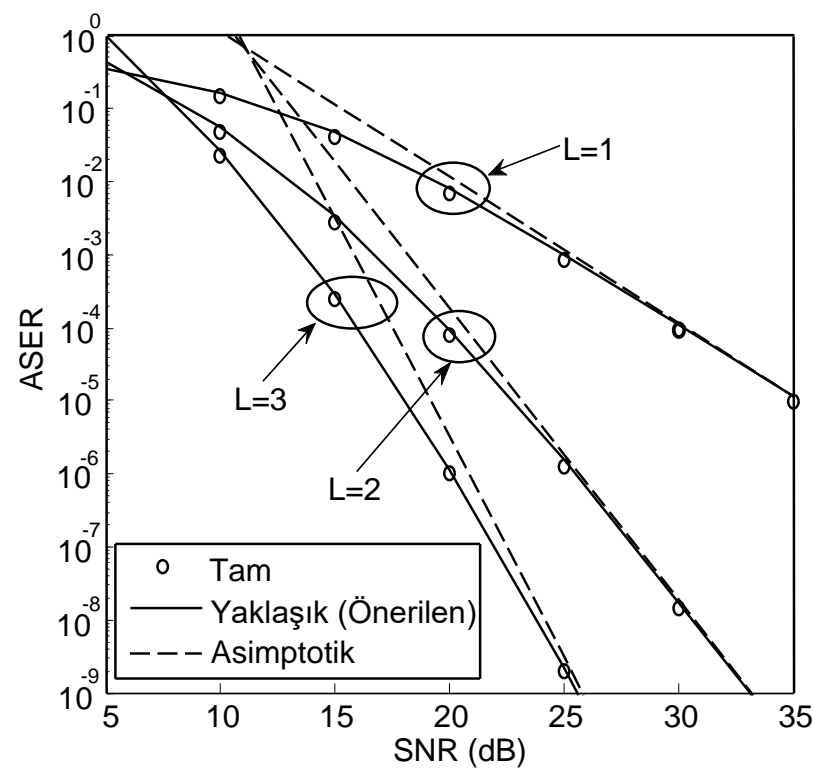

Şekil 2. 4X2 RQAM yöntemini kullanan $L$-kollu MRC alıc1lı sistemlerin Fisher-Snedecor F sönümlü kanallarda kol sayısına göre ASER performansı

Şekil 3'te, kullanılan modülasyon seviyesindeki değişimin ele alınan sistemin performansına etkisi incelenmiştir. Burada $m=3, m_{\mathrm{s}}=20, \beta=1$ ve kol sayısı $L=2$ olarak alınmıştır.

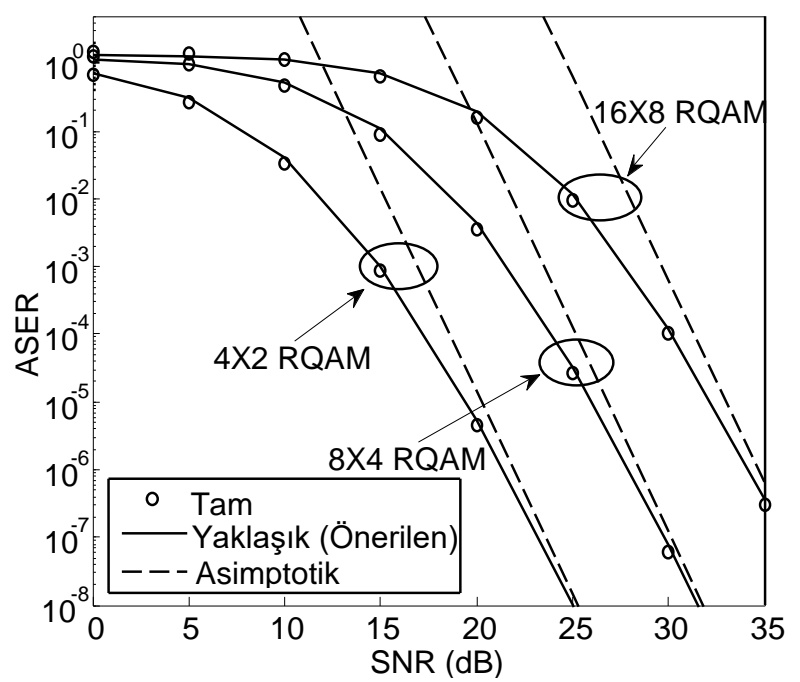

Şekil 3. Farklı RQAM modülasyon seviyeleri için $L$-kollu MRC alıc1lı sistemin Fisher-Snedecor F sönümlü kanallardaki ASER performans1

Modülasyon seviyeleri $4 \mathrm{X} 2,8 \mathrm{X} 4$ ve $16 \mathrm{X} 8$ olarak ele alınmış ve her bir seviye için sistemin performansı Şekil 3 'te sunulmuştur. $\mathrm{Bu}$ şekilden görüldüğü üzere, ele alınan sistemin performansı modülasyon seviyesi arttıkça kötüleşmektedir. Modülasyon seviyesi arttıkça iletilen sembol sayısı da arttığından beklenildiği gibi sistemin hata oranı da artmaktadır. Yine bu şekilden de açık bir biçimde yaklaşık ve tam sonuçların birbirine oldukça yakın olduğu, asimptotik sonuçların da yüksek SNR bölgesinde çok iyi uyum gösterdiği gözlenmektedir. $\mathrm{Bu}$ da önerilen ifadenin doğruluğunu ortaya koymaktadır.

Bu sonuçlara ek olarak, sönümlenme parametresinin $(m)$ $L$-kollu MRC alıcılı sistemin ASER performansına etkisi $m_{\mathrm{s}}=50, L=2$ ve $\beta=1$ durumunda $8 \mathrm{X} 4$ RQAM yöntemi kullanılarak Şekil 4'te sunulmuştur. Bu şekildeki sonuçlar $m$ parametresinin değeri 2.5 ve 3.5 şeklinde değiştirilerek elde edilmiştir.

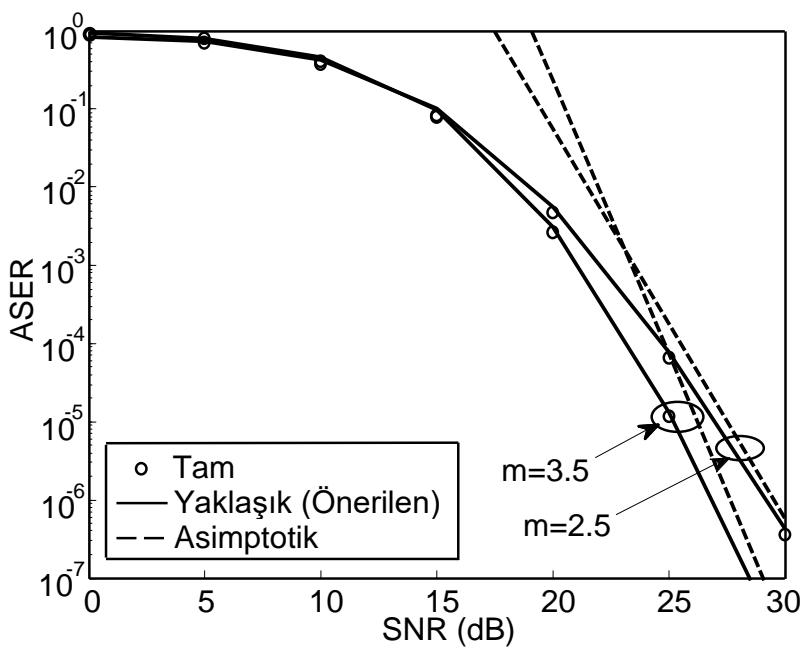

Şekil 4. Farklı sönümlenme parametresi değerleri için 8X4 RQAM kullanan $L$-kollu MRC alıcılı sistemin Fisher-Snedecor F sönümlü kanallardaki ASER performans1 
Sönümlenme parametresinin değerinin artması kanal şartlarının iyileşmesi anlamına geldiğinden beklenildiği gibi en iyi performansın $m=3.5$ durumunda oluştuğu görülmektedir. Şekil 2 ve Şekil 3'te olduğu gibi yine bu şekilde de yaklaşık ve tam sonuçların birbirine oldukça yakın uyum gösterdiği, asimptotik sonuçların ise yüksek SNR bölgesinde oldukça uyumlu olduğu gözlenmektedir.

\section{Sonuçlar}

Bu çalışmada, $L$-kollu MRC alıcısına sahip bir kablsouz haberleşme sisteminde RQAM yöntemi kullanıldığ durumda Fisher-Snedecor F sönümlü kanallardaki ASER performansı analiz edilmiştir. Gauss $Q$ fonksiyonunun oldukça iyi bir eksponansiyel yaklaşıklığı kullanılarak ele alınan sistemin ortalama sembol hata oranı için bir üst sınır ifadesi türetilmiştir. Türetilen bu ifade kullanılarak çeşitli sistem parametre değerleri için performans analizleri sunulmuştur. Buna ek olarak ele alınan sistem için asimptotik ASER ifadesi önerilmiştir. Yapılan analiz sonucunda önerilen ifadeden elde edilen sonuçların tam sonuçlara oldukça yakın olduğu ve iyi bir üst sınır elde edildiği ortaya konulmuştur. Ayrıca asimptotik sonuçların ise yüksek SNR bölgesinde diğer sonuçlar ile çok iyi uyum içinde olduğu gösterilmiştir.

\section{Çıkar çatışması}

Yazarlar çıkar çatışması olmadığını beyan etmektedir.

\section{Benzerlik oranı (iThenticate): $\% 13$}

\section{Kaynaklar}

[1] M. K. Simon and M-S. Alouini, Digital communication over fading channels, 2nd ed. Hoboken, New Jersey, USA: IEEE: John Wiley \&Sons, Inc., 2005.

[2] C. R. C. M. da Silva and M. D. Yacoub, A generalized solution for diversity combining techniques in fading channels, IEEE Transactions on Microwave Theory and Techniques, 50, 46-50, 2002. https://doi.org/10.1109/22.981244

[3] J. Beiranvand and H. Meghdadi, Analytical performance evaluation of MRC receivers in massive MIMO systems, IEEE Access, 6, 53226-53234, 2018. https://doi.org/10.1109/ACCESS.2018.2866795

[4] N. Kapucu, M. Bilim and I. Develi, A comprehensive performance analysis of relay-aided CDMA communications over dissimilar fading channels, AEÜ -International Journal of Electronics and Communications, 83, 339-347, 2018. https://doi.org/ 10.1016/j.aeue.2017.09.017

[5] D. Dixit and P. R. Sahu, Performance of $L$-branch MRC receiver in $\eta-\mu$ and $\kappa-\mu$ fading channels for QAM signals, IEEE Wireless Communications Letters, 1, 316-319, 2012. https://doi.org/10.1109/WCL.2012. 042512.120240

[6] P. Kumar and P. R. Sahu, Analysis of $M$-PSK with MRC receiver over $\kappa-\mu$ fading channels with outdated CSI, IEEE Wireless Communications Letters, 3, $557-$ 560, 2014. https://doi.org/10.1109/LWC.2014. 2355849
[7] A. D. Singh and R. Subadar, Capacity analysis of MRC receiver with adaptive transmitters over TWDP fading channels, 2015 International Symposium on Advanced Computing and Communication (ISACC), pp. 1-5, Silchar, India, Sept. 2015. https://doi.org/ 10.1109/ISACC.2015.7377357

[8] T. Liu, J. Tong, Q. Guo, J. Xi, Y. Yu and Z. Xiao, On the performance of massive MIMO systems with lowresolution ADCs and MRC receivers over Rician fading channels, IEEE Systems Journal, 2020. (Accepted for publication) https://doi.org/10.1109/ JSYST.2020.3014647

[9] S. K. Yoo, S. L. Cotton, P. C. Sofotasios, M. Matthaiou, M. Valkama and G. K. Karagiannidis, The FisherSnedecor $F$ distribution: a simple and accurate composite fading model, IEEE Communications Letters, 21, 1661-1664, 2017. https://doi.org/ 10.1109/LCOMM.2017.2687438

[10] H. Du, J. Zhang, K. P. Peppas, H. Zhao, B. Ai and X. Zhang, On the distribution of the ratio of products of Fisher-Snedecor $F$ random variables and its applications, IEEE Transactions on Vehicular Technology, 69, 1855-1866, 2020. https://doi.org/ 10.1109/TVT.2019.2961427

[11] N. Kapucu, Error performance of digital modulations over Fisher-Snedecor $F$ fading channels, AEÜ International Journal of Electronics and Communications, 108, 73-78, 2019. https://doi.org/ 10.1016/j.aeue.2019.06.005

[12] N. Kapucu and M. Bilim, Analysis of analytical capacity for Fisher-Snedecor $\mathrm{F}$ fading channels with different transmission schemes, Electronics Letters, 55, 283-285, 2019. https://doi.org/10.1049/el.2018.7813

[13] F. S. Almehmadi and O. S. Badarneh, On the effective capacity of Fisher-Snedecor F fading channels, Electronics Letters, 54, 1068-1070, 2018. https://doi.org/10.1049/el.2018.5479

[14] S. Chen, J. Zhang, G. K. Karagiannidis and B. Ai, Effective rate of MISO systems over Fisher-Snedecor $F$ fading channels, IEEE Communications Letters, 22, 2619-2622, 2018. https://doi.org/10.1109/LCOMM. 2018.2876426

[15] O. S. Badarneh, P. C. Sofotasios, S. Muhaidat, S. L. Cotton and D. B. da Costa, Product and ratio of product of Fisher-Snedecor F variates and their applications to performance evaluations of wireless communication systems. IEEE Access, 8, 215267-215286, 2020. https://doi.org/10.1109/ACCESS.2020.3039680

[16] H. Al-Hmood and H. S. Al-Raweshidy, Selection combining scheme over non-identically distributed Fisher-Snedecor $\mathrm{F}$ fading channels, IEEE Wireless Communications Letters, 10, 840-843, 2020. https://doi.org/10.1109/LWC.2020.3046519

[17] H. Shankar and A. Kansal, Performance analysis of MRC receiver over Fisher Snedecor $(F)$ composite fading channels, Wireless Personal Communications, 117, 1337-1359, 2021. https://doi.org/10.1007/ s11277- 020-07925-8 
[18] O. S. Badarneh, D. B. da Costa, P. C. Sofotasios, S. Muhaidat and S. L. Cotton, On the sum of FisherSnedecor $F$ variates and its application to maximalratio combining, IEEE Wireless Communications Letters, 7, 966-969, 2018. https://doi.org/10.1109/ LWC.2018.2836453

[19] R. Singh and M. Rawat, Physical layer security of MRC in Fisher-Snedecor $F$ fading channels, 2019 IEEE International Conference on Advanced Networks and Telecommunications Systems (ANTS), pp. 1-5, Goa, India, Dec. 2019. https://doi.org/10.1109/ ANTS47819. 2019.9118155

[20] S. K. Yoo, S. L. Cotton, P. C. Sofotasios, S. Muhaidat and G. K. Karagiannidis, Level crossing rate and average fade duration in $F$ composite fading channels, IEEE Wireless Communications Letters, 9, 281-284, 2020. https://doi.org/10.1109/LWC.2019.2952343

[21] P. Zhang, J. Zhang, K. P. Peppas, D. W. K. Ng and B. Ai, Dual-hop relaying communications over FisherSnedecor $F$-fading channels, IEEE Transactions on Communications, 68, 2695-2710, 2020. https://doi.org/10.1109/TCOMM.2020.2973263

[22] H. Du, J. Zhang, J. Cheng and B. Ai, Sum of FisherSnedecor $F$ random variables and its applications, IEEE
Open Journal of the Communications Society, 1, 342356, 2020. https://doi.org/10.1109/OJCOMS.2020. 2982770

[23] M. Bilim and N. Kapucu, Average symbol error rate analysis of QAM schemes over millimeter wave fluctuating two-ray fading channels, IEEE Access, 7, 105746-105754, 2019. https://doi.org/10.1109/ ACCESS.2019.2932147

[24] M. Chiani, D. Dardari and M. K. Simon, New exponential bounds and approximations for the computation of error probability in fading channels, IEEE Transactions on Wireless Communications, 2, 840-845, 2003. https://doi.org/10.1109/TWC.2003. 814350

[25] A. P. Prudnikov, Y. A. Brychkov and O. I. Marichev, Integrals and Series: More Special Functions, vol. 3. Gordon\&Breach Sci. Publ, NY, 1990.

[26] The Mathematical Functions Site. http://functions.wolfram.com, Accessed 27 May 2021.

[27] I. Gradshteyn and I. Ryzhik, Table of Integrals, Series and Products. 6th ed. New York, NY, USA: Academic, 2000 . 